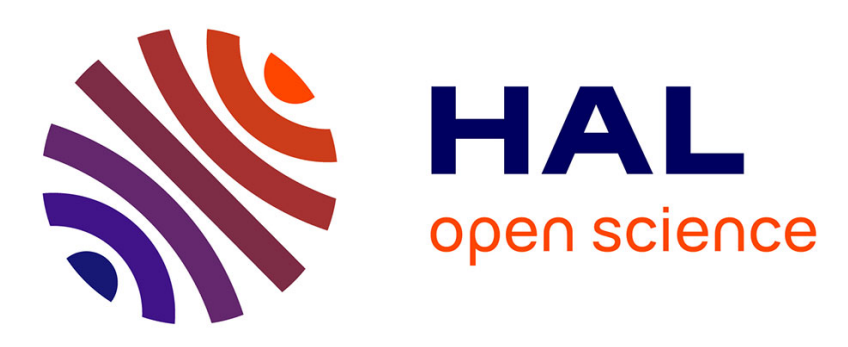

\title{
Extending satellite service availability through energy efficient cooperation
}

\author{
Riadh Dhaou, Benoît Escrig, Béatrice Paillassa, Caroline Bes
}

\section{To cite this version:}

Riadh Dhaou, Benoît Escrig, Béatrice Paillassa, Caroline Bes. Extending satellite service availability through energy efficient cooperation. 25th Annual IEEE International Symposium on Personal, Indoor and Mobile Radio Communications (PIMRC 2014), Sep 2014, Washington D.C., United States. pp. 1758-1762. hal-01394663

\section{HAL Id: hal-01394663 https://hal.science/hal-01394663}

Submitted on 9 Nov 2016

HAL is a multi-disciplinary open access archive for the deposit and dissemination of scientific research documents, whether they are published or not. The documents may come from teaching and research institutions in France or abroad, or from public or private research centers.
L'archive ouverte pluridisciplinaire HAL, est destinée au dépôt et à la diffusion de documents scientifiques de niveau recherche, publiés ou non, émanant des établissements d'enseignement et de recherche français ou étrangers, des laboratoires publics ou privés. 


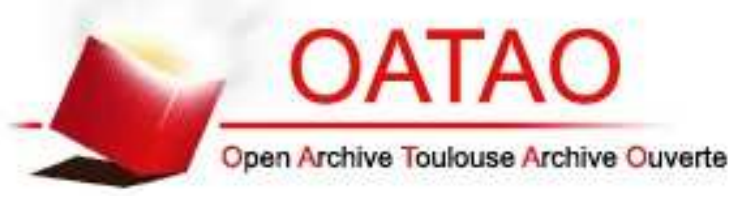

\section{Open Archive TOULOUSE Archive Ouverte (OATAO)}

OATAO is an open access repository that collects the work of Toulouse researchers and makes it freely available over the web where possible.

This is an author-deposited version published in : http://oatao.univ-toulouse.fr/ Eprints ID : 15184

The contribution was presented at PIMRC 2014:

http://www.ieee-pimrc.org/2014/

To cite this version : Dhaou, Riadh and Escrig, Benoît and Paillassa, Béatrice and Bes, Caroline Extending satellite service availability through energy efficient cooperation. (2015) In: 25th Annual IEEE International Symposium on Personal, Indoor and Mobile Radio Communications (PIMRC 2014), 2 September 2014 - 5 September 2014 (Washington D.C., United States).

Any correspondence concerning this service should be sent to the repository administrator: staff-oatao@listes-diff.inp-toulouse.fr 


\title{
Extending satellite service availability through energy efficient cooperation
}

\author{
Riadh Dhaou $^{(1)}$, Benoît Escrig ${ }^{(1)}$, Béatrice Paillassa ${ }^{(1)}$, Caroline Bès ${ }^{(2)}$ \\ (1) Université de Toulouse, INP/ENSEEIHT, IRIT, 2 rue Camichel, F-31000 Toulouse, France \\ E-mail : \{dhaou,escrig,paillassa\}@enseeiht.fr \\ (2) French Space Agency CNES, Toulouse, France \\ E-mail : caroline.bes@cnes.fr
}

\begin{abstract}
In this paper, we address the design of a cooperative protocol for a hybrid satellite/terrestrial emergency system. We want to perform energy savings compared to the case where all the terrestrial relay nodes are forwarding satellite messages to ground receivers. This is done via the selection of relevant relay nodes. The parameterization of the protocol phases has been done through simulations and takes into account the duration of the selection process, the number of selected nodes, and the signaling overhead. The selection process based on a node identifier (ID) appears to provide greater energy savings compared to the selection process based on the signal to interference and noise ratio (SINR). The solutions have been implemented in the real case scenario of forest fire that has been thoroughly documented by the US administration. According to the scenario parameters, $100 \%$ of the masked nodes are reached after cooperation.

Index Terms-satellite communications; hybrid systems; emergency communications; cooperative protocols; relay selection
\end{abstract}

\section{INTRODUCTION}

Satellite Communications (SCs) are known to provide services over large coverage areas without the need of a ground infrastructure [1]. This benefit is paramount in case of a natural disaster (tsunami, earthquake) when rescue teams must be deployed over devastated areas [2] [3] [4]. However, the availability of a satellite data service is limited by the masking effect when there are obstacles between the satellite and the ground receivers, i.e., when the received signal to interference and noise ratio (SINR) is too low to achieve reliable decoding of the satellite message.

Diversity techniques such as time, frequency, and satellite diversity techniques [5] can tackle this problem by providing ground receivers with different versions of a satellite signal. ${ }^{1}$ In the context of hybrid satellite/terrestrial systems, cooperative communications can also enhance the service availability by allowing relay nodes to forward satellite messages to masked nodes [6] [7] [8].

Cooperation schemes have already been implemented in broadcasting systems such as Digital Video Broadcasting Satellite Handheld (DVB-SH) by means of fixed relays, called gap-fillers. But these relays cannot handle dynamic situations, i.e., situations when ground receivers are mobile nodes [9]. On

\footnotetext{
${ }^{1}$ Increasing the number of versions reduces the probability that all the versions exhibit a low SINR at the same time.
}

the contrary, it has been shown that non masked (NoM) mobile nodes can serve as relay nodes in a dynamic environment. ${ }^{2}$

For instance, in [6], satellite downlink (DL) communications are divided into two time-slots. During the first time-slot, the satellite is transmitting its message toward ground receivers. During the second time slot, the NoM nodes broadcast the satellite message. ${ }^{3}$ Hence, the masked nodes are given a second opportunity to get the satellite message. No extra signaling is needed here to set the cooperation. No extra infrastructure, such as gap-fillers, is required. However, simplicity comes at the price of extra energy consumption. A masked node may receive two or more versions of the satellite signal even though only one version is needed. Moreover, an NoM node may broadcast the satellite message even if it is not in the range of a masked node.

The main contribution of the paper consists in designing a cooperative transmission scheme for hybrid satellite/terrestrial systems that both (i) increases the service availability, by tackling the masked node problem, and (ii) allows for some energy savings compared to the case when all NoM nodes serve as relay nodes, by selecting the best relay for each masked node. A second contribution consists in evaluating the proposed solutions on a real case. The context corresponds to a forest fire that has been investigated in [10].

The rest of the paper is organized as follows. The second section presents the wildfire scenario. The third section describes the protocol and the relay selection procedures. The fourth section addresses the simulation results and we conclude in section five.

\section{WILDFIRE SCENARIO}

A hybrid terrestrial/satellite system is implemented in the context of a wildfire. SCs are established between a remote operations center and emergency workers (firefighters, foresters) that are deployed on the fireground. The idea here is to propose a cooperation protocol between ground terminals so that masked nodes could receive the information from the

\footnotetext{
${ }^{2} \mathrm{NoM}$ nodes are the nodes that have successfully decoded the satellite message.

${ }^{3} \mathrm{We}$ assume that not all the ground receivers are masked. This assumption makes sense since the sizes of obstacles are small compared to the sizes of the coverage areas.
} 
operations center via NoM nodes. The terrain is so steep that no fire trucks with satellite transmission equipment can access the area.

The scenario is based on a real case that has been thoroughly documented by the U.S. Department of Agriculture [10]: a forest fire in a mountain area. In particular, the investigation showed that better communication between the fire control center and firefighters would have lead to a reduced number of deaths during the incident.

The DL traffic is constituted by images/maps transmissions (CBR $500 \mathrm{~KB}$ every 10 minutes). For sake of simplicity, the uplink (UL) traffic, and hence voice calls, will not be considered in this paper.

Twenty-one half-duplex nodes are deployed on the fireground (a first group of seven nodes and an additional group of fourteen nodes). Nodes are able to transmit on both a satellite link and a terrestrial WIFI link. Cooperation takes place in adhoc mode, i.e., there is no fixed relays. Emergency workers are deployed on a 400 hectares area (1000 meters x 4000 meters) and the deployment follows the fire line [10] according to three concentric and elliptic areas $\left(Z_{1}, Z_{2}\right.$, and $\left.Z_{3}\right)$ depicted in Fig.1.

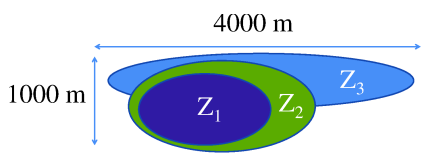

Fig. 1. Fire spread diagram according to [10].

In the context of a forest environment, propagation models comprise two components: a plane earth path loss component and an additional component that varies according to studies, contexts, forest types, and frequency ranges. We have selected the Fitted ITU-R model developed in [11] [12]. The multipath fading margin is neglected here but multipath effects are taken into account by using OFDM (Orthogonal Frequency Division Multiplex) waveforms.

\section{PROTOCOL}

The proposed protocol is called OHR for One Hop Relay. The time axis for the transmission of one frame is divided into two time-slots. The first time-slot (TS1) is for the satellite transmission. Data and control frames are transmitted during the second time-slot (TS2) in order to detect and select relay nodes, and to forward the information transmitted by the satellite (see Fig.2).

During the detection step, all NoM nodes that have successfully decoded the satellite message, send a detection (DET) frame. The same frame is used for all nodes. The NoM nodes are considered as potential relays or relay candidates. Nodes that have not received the message from the satellite, but have successfully decoded the DET frame, detect themselves as being masked nodes. Considering OFDM signals on the terrestrial segment, DET frames from different NoM nodes are arriving at masked nodes within the OFDM guard interval, so they can be combined constructively. Basically, the

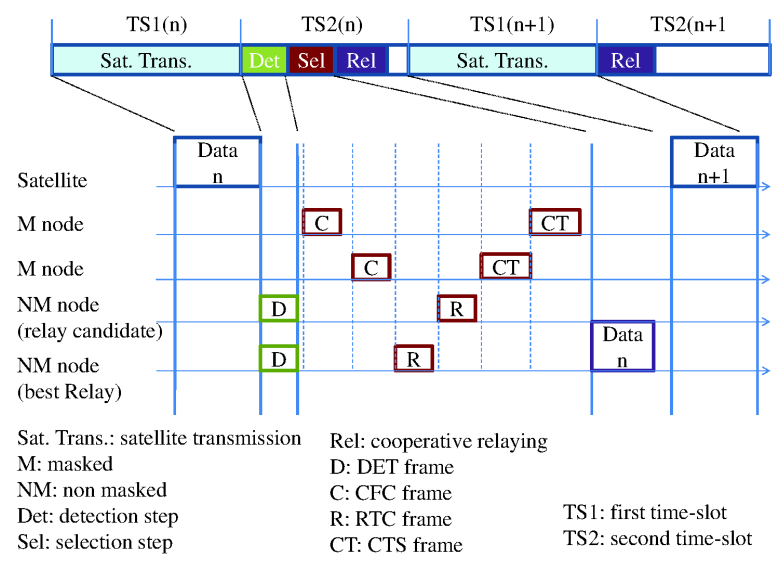

Fig. 2. Frame exchange sequence in the OHR protocol.

protocol combines OFDM signals in the contention free steps, i.e., during the detection step and cooperative relaying step. Conversely, the selection step uses a contention based WIFI access.

During the selection step, each masked node aims at selecting its best relay among the NoM nodes in its range. Limiting the number of relays reduces considerably the energy consumption caused by data forwarding. When a masked node successfully decodes the DET frame, it sends a CFC frame (Call For Cooperation) to initiate the selection process. The CFC frames are transmitted after a random timout in order to minimize the risk of a collision. In case of a collision, the masked nodes wait for a random duration before trying to access the channel again. At that point, NoM nodes know that they have to help masked nodes. We now proceed to the competition step.

Potential relays are evaluated according to a given metric. In this paper, we compare two different metrics. The first metric is based on a node identifier (ID). The best relay corresponds to the node with the lowest address. The second metric is based on an SINR measurement. The best relay corresponds to the node that exhibits the highest SINR at the masked node. The metrics are collected by masked nodes through RTC messages (Ready To Cooperate) sent by the potential relays. Each relay candidate triggers a timer upon receiving the CFC frame. When the timer expires, an RTC frame is sent. So, when a node sends an RTC frame, it signals its presence to masked nodes and indicates its willingness to cooperate. Hence, masked nodes get a knowledge of their neighboring nodes and can select their best relay.

Note that the RTC frame of the NoM node $N_{i}$ contains its ID when the ID metric is used. When the SINR metric is considered, $N_{i}$ send the SINR values corresponding to each received $\mathrm{CFC}$ frame, i.e, the SINR values between itself and all the masked nodes in its range that sent a CFC frame. We can go a step further. An RTC frame of an NoM node $N_{i}$ could contain the list of all the masked nodes in the range of $N_{i}$ and 
their corresponding metrics. When the RTC frame from $N_{i}$ is overheard by another potential relay $N_{j}$, this node could waive its participation to the selection process as soon as it discovers that $N_{i}$ has better metrics with respect to the masked nodes $N_{j}$ is about to help. This optimization has been considered and evaluated. It results that the duration of the cooperation set-up and the signaling overhead increased dramatically, thus precluding the implementation of such an approach. Due to the lack of space, the corresponding results will not be reported here.

We now address the way timers are set. Timer values depend on the metric. When the selection is based on the lowest ID (resp. highest SINR), timer values are proportional (resp. inversely proportional) to the metric [13] [14]. So the best relay always signals its presence first. This contributes to minimize the duration of the distributed selection process. When IDs are used, timers can be configured so that there are no collision between different RTC frames. For instance, a timer $\tau_{i}$ associated to node $N_{i}$ can be computed using the formula $\tau_{i}=\sum_{k=1}^{N} a_{i k} \times \tau_{k}$ where the $a_{i k}$ denote the hexadecimal digits of the MAC address of $N_{i}$ and $\tau_{k}$ denotes the time increment associated to the $k^{\text {th }}$ digit. When the SINRs are used, there is a risk of a collision since the metrics belong to the set of real numbers. In the case of a collision, NoM nodes access the channel later by triggering a timer with a random duration. Once the masked nodes have collected all the CFC frames, they select the best relay. The result of the selection step is transmitted in a CTS (Clear To Send) control frame that contains the identifier of the best relay. Here also, when a collision occurs, the masked nodes wait for a random duration before trying to access the channel again. In this paper, the values of the timers and the duration of the selection step are adjusted by simulation experiments based on the studied scenarios.

During the relaying step, NoM nodes are forwarding the satellite message. Since the signals are to be combined at the masked terminals, the forwarding is performed using the same modulation and coding scheme. There is no adaptive modulation and coding (AMC) mechanism. Hence, the energy savings provided by the SINR-based method are limited since it is not possible to transmit the message using a higher order modulation. This constraint limits the interest of selecting the best relay based on the SINR criterion. We assume that the relay terminals are synchronized, so they all transmit quasi-simultaneously and the forwarded signals can be added constructively at the receiver using a maximum ratio combiner. Terrestrial signals are not combined with the satellite one, since masked nodes did not receive a usable signal. When the node speed is low, the detection and selection phases can be omitted for subsequent frames (see Fig.2).

Note also that the cooperative transmission scheme can be combined with a repetition scheme from the satellite. We consider that a single repetition scheme from the satellite is not enough to address any shadowing profile, any mobility model. The service availability is increased here through the help of surrounding nodes.
TABLE I

DURATION OF THE PHASES IN SECONDS

\begin{tabular}{|c|c|c|}
\hline Detection & Selection & Cooperation \\
\hline 0.01 & 0.03 & 0.06 \\
\hline
\end{tabular}

\section{Protocol REFINEMENTS THROUGH SIMULATIONS}

The choice for a simulation-based approach comes from the fact that there is no fully satisfactory mathematical solutions to our problem. For instance, minimizing the duration of the timer-based selection process leads to timers with smaller values. This increases the probability of a collision between relay candidates when random timers are used. That, in turns, increases the duration of the selection process [14]. So, instead of considering all possible options, we present here simulations results to attest the feasibility of the proposed solution.

The implementation of the proposed protocol is performed under the ns- 2 network simulator. The purpose of the simulations is to refine the design of the protocols. Simulations aim to evaluate the duration of the protocol phases (detection, selection, and cooperation), the performance of the OHR protocol in terms of number of potential relays, number of selected relays according to the selection criterion: lowest ID or maximum SINR. The validation step of the protocol requires the development of specific modules in ns-2: a new propagation model (Fitted ITU-R) adapted to the forest environment, a technique for the coherent combining of multiple signals, two selection methods based on the lowest ID or the maximum SINR.

In the simulated scenarios, the terminals are placed on an ellipse. A given percentage of nodes is masked. The choice of masked nodes is different from one scenario to another: either the masked nodes are randomly selected, or an entire geographical area is shadowed. To synchronize the nodes and separate the protocol steps, each protocol phase has a specific duration. The values of these durations have been adjusted so the risk of collision is minimized during the cooperation setup and have been determined by ns- 2 simulations. The results are depicted in Table I. These results have been obtained for a scenario in which the duration of the DL time-slot is $0.1 \mathrm{~s}$, so the total duration of the three-phase protocol is also $0.1 \mathrm{~s}$.

Several parameters have been evaluated in the simulations, including the duration of the cooperation set-up, i.e., the average time for a masked node to establish a cooperation, and the percentage of lost packets. These parameters have been estimated based on the density of the nodes, i.e., the total number of nodes in a specific geographical area, and according to the transmitted power. Each parameter has been evaluated taking into account the two relay selection methods, and the percentage of masked nodes, $25 \%, 50 \%$ and $75 \%$ of the total number of nodes, with a random spatial distribution.

\section{A. Cooperation set-up time}

First, we vary the node density and we plot the time required to establish cooperation for different percentages of masked nodes. The results are depicted in Fig.3. Since the intervention 
zone has a fixed surface, the parameter "Density" in the figures depicts the number of nodes in the intervention zone. In Fig.3, the set-up duration converges rapidly to $1.25 \mathrm{~s}$ whatever is the selection method (ID or SINR). So we choose to set the maximal duration for the selection step to $1.5 \mathrm{~s}$. Moreover, the transmission power is set so that the node graph is a connected graph, i.e., there is at least one path between any two nodes. This minimizes the probability of a set-up fail.
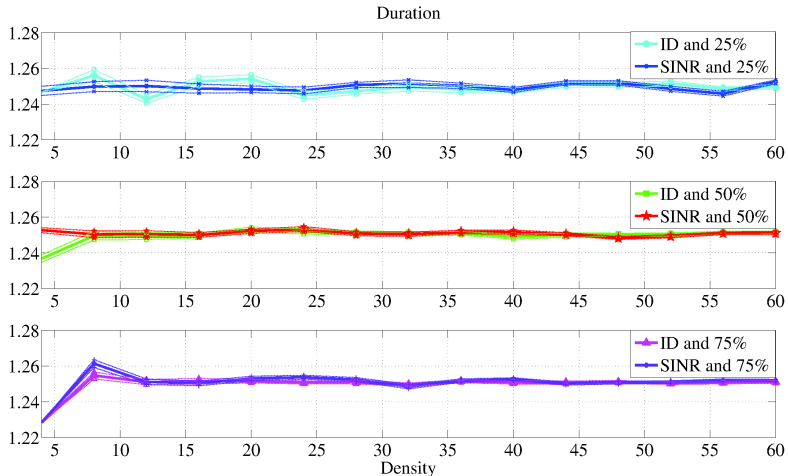

Fig. 3. Cooperation set-up duration for the OHR protocol. Mean values are represented by bold lines, minimum and maximum values by dotted lines. Labels ID and SINR denote the selection methods. The percentages represent the percentages of masked nodes.

\section{B. Choice of the selection method}

First, the overhead induced by the selection step is investigated (see Fig.4 and Fig.5). Here also, the parameter "Density" depicts the number of nodes in the intervention zone. Since there is no great difference between minimum/maximum values and the mean values, only mean value curves are plotted in the following figures. We note that the overhead decreases with the percentage of masked terminals. The total number of transmitted bits increases with the density of nodes, irrespective of the selection mode, ID or SINR.

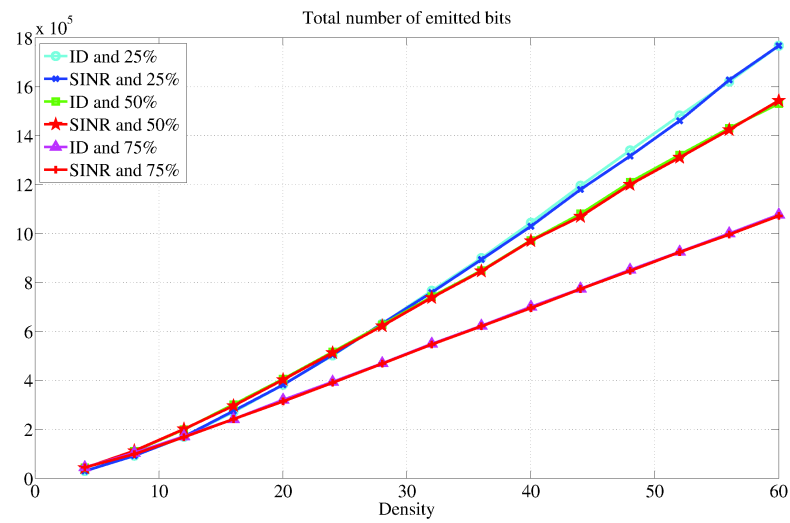

Fig. 4. Mean values for the total number of emitted bits during the selection phase for the OHR protocol. Labels ID and SINR denote the selection methods. The percentages represent the percentages of masked nodes.

Second, the selection methods have been evaluated according to the number of selected relays. Fig. 6 shows the number

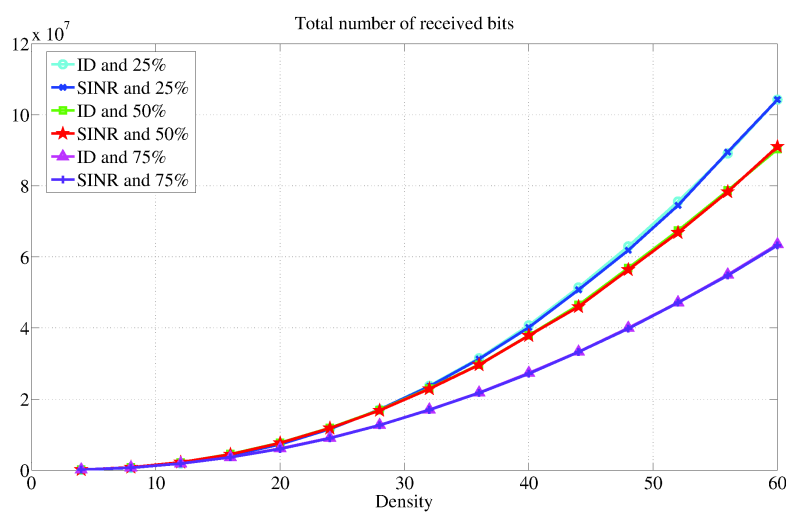

Fig. 5. Mean values for the total number of received bits during the selection phase for the OHR protocol. Labels ID and SINR denote the selection methods. The percentages represent the percentages of masked nodes.

of selected relays as a percentage of the number of detected NoM nodes. The percentages are plotted as a function of the node density.

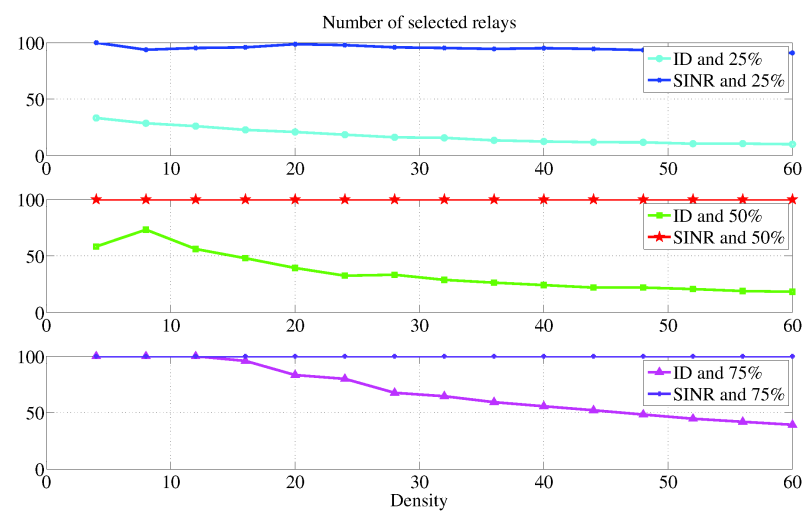

Fig. 6. Mean values for the number of selected relays as a percentage of detected relays for the OHR protocol. Labels ID and SINR denote the selection methods. The percentages represent the percentages of masked nodes.

We notice a clear difference between the lowest ID and the maximum SINR methods. When the number of nodes is increasing, it is always possible to find a best relay according to the SINR criterion. So the number of selected relays is increasing when the SINR criterion method is used. On the contrary, when the ID solution is used and the number of nodes is increasing, the probability to find a relay with the lowest ID approach increases with the density, leading the a decreased number of selected relays, and hence to a lower energy consumption.

Several other outcomes were observed, which are rather straightforward:

- When the number of masked nodes increases, the time required for all nodes to be able to cooperate increases.

- The SINR increases as the density of nodes increases.

- The percentage of potential relay decreases with the density of terminals.

- Conversely, the percentage of selected relay tends to decrease when the density of terminals increases. 
More importantly, according to the forest fire scenario, all the masked nodes receive the satellite message after the cooperation step.

At first glance, we can say that the selection mechanism based on the SINR provides a better SINR on each link but the energy consumption at the network level is higher because there is potentially one single best relay for each masked node. On the contrary, the SINR on each link is not minimized when best relays are selected according to the lowest ID. However, the energy consumption is lower since one single NoM node can serve several masked nodes. We can go a step further using the following example.

Note first that, in all cases, all the relays are using the same waveform, i.e., the same modulation and coding scheme (MCS). The selected MCS is the one that provides the best robustness to channel impairments. We analyze the scenario presented in Fig.7 where $M_{1}$ and $M_{2}$ are two masked nodes and $R_{1}$ and $R_{2}$ are two potential relays. We assume that $M_{1}$ and $M_{2}$ are in the range of both $R_{1}$ and $R_{2}$. When the IDbased solution is considered, one relay can serve both $M_{1}$ and $M_{2}$. Let $R_{2}$ be the selected relay. When $R_{1}$ is closer to $M_{1}$ than $R_{2}$, one can claim that, in the case of the SINR-based selection, the transmission between $R_{1}$ and $M_{1}$ could use a little less energy than in the case of the ID-based solution. However, in the case of the SINR-based solution, we also must take into account that two transmissions will take place instead of one. So the energy saving that is earned on the $R_{1}$ $M_{1}$ link is counterbalanced by the fact that two transmissions must take place, the one from $R_{1}$ to $M_{1}$ and the one from $R_{2}$ to $M_{2}$. That leads us to select the ID-based solution.

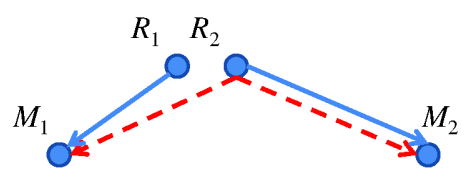

Fig. 7. Relay transmissions in the case of the ID-based selection (dotted line) and the SINR-based selection (solid line).

\section{CONCLUSION}

SCs provide a relevant contribution to emergency systems since they allow the provision of data services over wide coverage areas. In this paper, we propose a cooperative hybrid satellite/terrestrial system in the context of a forest fire. The satellite segment is used in a first time-slot to broadcast emergency data. During a second-time slot, relay nodes are selected among the NoM nodes in the neighborhood of masked nodes. Then the selected nodes forward the satellite message they have received. The issue is to perform some energy savings compared to the case where all the NoM nodes forward the satellite messages to their neighboring nodes. For that purpose, two selection mechanisms have been proposed: one is based on the lowest ID and the other one is based on the maximum SINR.
From the simulation results, it appears that the ID-based selection process provides greater energy savings than the one based on the SINR. Even if the SINR is not maximized for all wireless links, this is largely counterbalanced by the fact that a relay can serve several masked nodes. The second contribution of the paper consists of applying the proposed protocol to the real case scenario of a forest fire. In the specific case that has been studied in this paper, the satellite messages could be delivered to $100 \%$ of the masked nodes after a cooperation step.

Further studies could address the case of multi-hop cooperation. Masked nodes, once they have received the satellite message from NoM nodes, could in their turn forward the message. Furthermore, fixed nodes have been considered in this paper. The case of mobile nodes should also be addressed to tackle more compelling issues and a special attention should be paid to mobility models. Lastly, synchronization at the signal level is also a major issue. This is left for future work.

\section{REFERENCES}

[1] P. Chini, G. Giambene, and S. Kota, "A survey on mobile satellite systems," Int. J. Sat. Commun., vol. 28, pp. 29-57, 2009.

[2] E. Del Re, S. Jayousi, S. Morosi, L. Ronga, M. De Sanctis, E. Cianca, E. Ruggieri, M. Falletti, A. Iera, G. Araniti, and C. Sacchi, "Salice satellite-assisted localization and communication systems for emergency services," IEEE A \& E Systems Magazine, pp. 4-15, september 2013.

[3] B. Evans, M. Werner, E. Lutz, M. Bousquet, G. Corazza, G. Maral, and R. Rumeau, "Integration of satellite and terrestrial systems in future multimedia communications," IEEE Transactions on Wireless Communications, vol. 12, no. 5, pp. 72-80, october 2005.

[4] G. Iapichino, C. Bonnet, O. del Rio Herrero, C. Baudoin, and I. Buret, "Advanced hybrid satellite and terrestrial system architecture for emergency mobile communications," in AIAA International Communications Satellite Systems Conference, june 2008, pp. 1-8.

[5] G. Cocco, C. Ibars, and O. del Rio Herrero, "Cooperative satellite to land mobile gap-filler-less interactive system architecture," in 5th Advanced satellite multimedia systems conference and the 11th signal processing for space communications workshop, september 2010, pp. 309-314.

[6] B. Paillassa, B. Escrig, R. Dhaou, M.-L. Boucheret, and C. Bes, "Improving satellite services with cooperative communications," International Journal of Satellite Communications and Networking, vol. 29, no. issue 6, pp. 479-500, december 2011.

[7] D.-S. Ahn, S. Kim, H. W. Kim, and D.-C. Park, "A cooperative transmit diversity scheme for mobile satellite broadcasting systems." International Journal on Satellite Communications and Networking, vol. 28, pp. 352-368, 2010.

[8] S. Kim, "Evaluation of cooperative techniques for hybrid/integrated satellite systems," in IEEE International Conference on Communications, june 2011, pp. 1-5.

[9] ETSI, "Digital video broadcasting (dvb)," ETSI, Tech. Rep., 2008.

[10] "Thirtymile Fire Investigation Report," United States Department of Agriculture, Forest Service, Tech. Rep., 2001.

[11] M. O. Al-Nuaimi and R. B. L. Stephens, "Measurements and prediction model optimization for signal attenuation in vegetation media at centimetre wave frequencies," IEE Proceedings in Microwave Antennas Propagation, vol. 145, pp. 201-206, june 1998.

[12] Y. S. Meng, Y. H. Lee, and B. C. Ng, "Empirical near ground path loss modeling in a forest at vhf and uhf bands," IEEE Transactions on Antennas and Propagation, vol. 57, no. 5, pp. 1461-1468, may 2009.

[13] B. Escrig, "On-Demand Cooperation MAC Protocols with Optimal Diversity-Multiplexing Tradeoff," in Proc. IEEE International Conference on Wireless Communications and Networking Conference (WCNC), 2010.

[14] A. Bletsas, A. Khisti, D. P. Reed, and A. Lippman, "A Simple Cooperative Diversity Method Based on Network Path Selection," IEEE Journal on Selected Areas in Communications, vol. 24, no. 3, pp. 659-672, 2006. 